\title{
Current state of evidence of cannabis utilization for treatment of autism spectrum disorders
}

\author{
Rumi Agarwal ${ }^{1,2,3}$, Shanna L. Burke ${ }^{2,4^{*}}$ (i) and Marlaina Maddux ${ }^{1,2,5}$
}

\begin{abstract}
The core symptoms and co-morbidities associated with autism spectrum disorders (ASD) affect daily living and quality of life. Existing pharmacological interventions are only able to attenuate some related symptoms but are unable to address the underlying etiologies associated with ASD. Anecdotal evidence, which claims benefit from the use of cannabis to treat symptoms among this population, has been gaining popularity as families seek solutions.

This paper analyzed recent peer-reviewed literature to identify the current state of evidence regarding cannabis use for the ASD population. Systematic reviews, reports, and experimental studies were assessed to understand the current extent and nature of the evidence on the risks and benefits of cannabis use for ASD. At this time, three large-scale clinical trials are currently at varying stages of progress and publication of results. Only five small studies were identified that have specifically examined cannabis use in ASD. Given the sparse state of evidence directly assessed in this population, studies which examined effects of cannabis on shared pathological symptoms of ASD such as hyperactivity, sleep disorders, self-injury, anxiety, behavioral problems, and communication were also reviewed.

Studies revealed mixed and inconclusive findings of cannabis effects for all conditions, except epilepsy. Adverse outcomes were also reported, which included severe psychosis, increased agitation, somnolence, decreased appetite, and irritability. In addition, a wide range of cannabis compositions and dosage were identified within the studies, which impact generalizability.

There is currently insufficient evidence for cannabis use in ASD, which creates an urgent need for additional largescale controlled studies to increase understanding of risks and benefits and also to examine the impact of "entourage effects." This will support discussions of treatment options between health care providers and ASD patients and their families. Evidence may lead to a desired new line of treatment or prevent adverse outcomes from unsubstantiated use amongst families aiming for symptom reduction.
\end{abstract}

Keywords: Autism spectrum disorders, Autism, Cannabis, Cannabinoids

\footnotetext{
* Correspondence: sburke@fiu.edu

${ }^{2}$ BRAINN Lab, Florida International University School of Social Work, 11200

SW 8th Street, Miami, FL 33199, USA

${ }^{4}$ Florida International University School of Social Work Robert Stempel

College of Public Health and Social Work, 11200 S.W. 8th Street, AHC5 585,

Miami, FL 33199, USA

Full list of author information is available at the end of the article
}

(c) The Author(s). 2019 Open Access This article is distributed under the terms of the Creative Commons Attribution 4.0 International License (http://creativecommons.org/licenses/by/4.0/), which permits unrestricted use, distribution, and reproduction in any medium, provided you give appropriate credit to the original author(s) and the source, provide a link to the Creative Commons license, and indicate if changes were made. The Creative Commons Public Domain Dedication waiver (http://creativecommons.org/publicdomain/zero/1.0/) applies to the data made available in this article, unless otherwise stated. 


\section{Background}

Cannabis is derived from Cannabis sativa, one of the world's oldest propagated plants. Beginning in the nineteenth century, medical practitioners began to experiment with cannabis to treat tetanus, convulsive diseases, and mental disorders [1, 2] and later, cannabis extracts were available for purchase at physicians' offices and pharmacies, in America and Europe, to treat ailments such as stomach aches, migraines, and insomnia [3].

Today, however, cannabis, which is also commonly referred to as marijuana, remains illegal under federal law in the United States and is categorized as a schedule 1 drug under the Controlled Substances Act. At the state level, cannabis for medical purposes has been decriminalized in over 34 states [4], although physicians remain hesitant in recommending its use given the sparse state of evidence regarding its efficacy to treat specific conditions [5].

Hence, a conflicting spiral exists. Without scientific evidence to establish efficacy, cannabis as a potential course of treatment is often not recommended by practitioners. In turn, until the status of cannabis is changed from a schedule 1 drug, research on the potential uses of marijuana and its components is greatly inhibited [5].

\section{Medicinal uses of marijuana compounds}

The cannabis plant comprises numerous active chemical compounds, which include cannabinoids, terpenoids, and flavonoids. Two cannabinoids include cannabidiol (CBD) and $\triangle$ 9-tetrahydrocannabinol (THC) [6]. THC is the compound shown to have intoxicating effects and targets the endocannabinoid system in the central nervous system. It affects appetite, cognitive function, memory, and anxiety. CBD on the other hand, is thought to be anti-inflammatory, treat epilepsy and psychiatric disorders, and does so without the intoxicating side effects $[7,8]$.

Although extensive literature exists on the major cannabinoids $\mathrm{CBD}$ and $\mathrm{THC}$, interest has been increasing in other phytotherapeutic compounds of the cannabis plant, specifically, terpenoids. Terpenoids are the fragrant oils, which are naturally present in many plants, and over 200 have been reported. Examples of these include phytol, limonene, nerolidol, myrcene, caryophyllene oxidate, pinene, $\beta$ caryophyllene, and linalool. These terpenoids are Generally Recognized as Safe as food additives by regulatory bodies, including the US Food and Drug Administration (FDA), and the Food and Extract Manufacturers Association [6]. Studies in animals and humans have shown the medicinal effects of terpenes' [9] which demonstrate "anti-inflammatory, antioxidant, analgesic, anticonvulsive, antidepressant, anxiolytic, anticancer, antitumor, neuroprotective, anti-mutagenic, antiallergic, antibiotic and anti-diabetic attributes" [10]. It is suggested that cannabinoids and terpenes have a combined effect by "working synergistically" with each other. This interaction between the compounds of the cannabis plant is referred to as the "entourage effects," which have implications on the strains of cannabis which are bred to best treat individual symptoms and diseases $[9,11]$.

The marijuana plant, however, has not been approved by the FDA for the treatment of any health conditions. Some of its cannabinoids such as CBD, THC, or similar synthetic substances, however, have been approved for specific health issues [12]. At this time, the FDA has approved four drugs with cannabinoids. Epidiolex was approved in 2018 and contains CBD derived from the marijuana plant. It is an oral solution used to treat seizures associated with two rare, severe forms of epilepsy. In addition, Dronabinol and Nabilone, derived from synthetic cannabinoids were approved to treat nausea and vomiting resulting from chemotherapy. Dronabinol is a synthetic form of $\mathrm{THC}$ and can be used to aide patients in patients with appetite and weight loss secondary to AIDS. A liquid form of dronabinol, Syndros, was approved in 2016 by the FDA [12]. These medications may offer patients with these ailments an alternative treatment and relief from pain, at a time when a substitute for opioids is crucial [4]. Unfortunately, however, the rising popularity of medical cannabis poses challenges, as evidence to support the use of medical cannabis across many health conditions has been sparse [5].

Based upon the 2018 report from the National Academies of Sciences, Engineering and Medicine (a systematic review of existing studies) strong evidence was reported of the positive effect of cannabis for individuals diagnosed with multiple sclerosis and chronic pain and spasticity, nausea and vomiting due to chemotherapy, and individuals experiencing seizures $[13,14]$. However, the report also highlighted sparse or absent evidence supporting medical cannabis for many morbidities, including neurodevelopmental conditions [13, 14], such as autism spectrum disorders (ASD).

The prevalence of ASD has increased and 1 in every 59 children is estimated to be affected [15]. Symptoms such as motor impairment, anxiety, abnormal behavior, sleep problems, and epilepsy substantially impact the quality of life for these individuals [16]. Currently, pharmacologicalbased treatments attenuate some ASD symptomology, but do not address its underlying etiology [17] although research to examine future pharmacological options is ongoing, such as the interaction of oxytocin and its possible implication in improving social behavior [18].

In the meantime, families of children with ASD are reportedly making cannabis-related decisions based on the plethora of anecdotal evidence on the success of CBD to treat ASD related symptoms and comorbidities [19]. Given the need for additional studies on the effect of cannabis use and the possibility of alleviating symptoms of ASD that substantially interfere with day-to-day work, play, and comfort, it is necessary to review the current state of evidence from human studies to assess the risks 
and benefits of medical cannabis use amongst this vulnerable population. This will allow positive findings to be noted, highlight adverse health outcomes [20], and consequently identify pathways for future clinical studies.

\section{The endocannabinoid system}

The endocannabinoid system (ECS) is comprised of Gprotein coupled cannabinoid 1 (CB1R) and 2 (CB2R) receptors, endogenous bioactive lipid signals (endocannabinoids; eCBs), and both synthetic and metabolizing enzymes [21]. The ECS plays an important role in cannabinergic signaling of human health and disease [22]. The manipulation of eCBs offers therapeutic potential in the treatment and management of a wide range of conditions of the central nervous system, including, but not limited to psychiatric, neurodegenerative, and neuroinflammatory disorders [21, 22].

ASD models in mice have been useful in assessing alterations in the ECS. For instance, Fragile X Mental Retardation (FMR1) knockout mice show core symptoms that are relevant in studies of ASD, including social interaction deficits, repetitive behaviors, and hyperactivity [23-25]. Researchers have identified that alterations in the ECS may be linked to the ASD-like symptoms displayed in the FMR1 model [23, 24, 26]. This points to a potential intervention pathway through modification of ECS signaling, which has shown preliminary success in mouse models in decreasing anxiety and behavioral symptoms, increasing cognitive performance, and attenuating motor deficits.

Numerous bioactive eCBs have been identified, with the most active including Anandamide (AEA) and 2-arachidonoylglycerol (2-AG) [22]. The synthesis of these eCBs occurs via many pathways, including $\mathrm{Ca}^{2+}$-dependent $\mathrm{N}$-acyltransferase and $\mathrm{N}$ acylphosphatidylethanolamine-hydrolyzing phospholipase D and diacylglycerol lipase (DAGL) and phospholipase $C \beta$ respectively $[21,27]$. AEA and 2AG are then broken down by fatty acid amide hydrolase (FAAH), and monoacylglycerol lipase (MAGL) [21]. BTBR mice are also used as nongenetic ASD models given that they display anatomical features that are consistent with ASD models [28]. In addition, these mice demonstrate deficits in play, social behavior $[29,30]$, repetitive behaviors [30, 31], cognitive impairments [32], and excess blood levels of corticosterone in the presence of stressful stimuli [33]. In the BTBR model, increasing AEA levels acute administration of URB597 increased AEA levels, which sunsequently reversed social deficits, though additional studies are needed to investigate altering the ECS in BTBR models [18].
The nature of the ECS is vast and complex, with AEA and 2-AG representing only a couple of the possible eCBs and each having multiple associated pathways of synthesis and enzymatic degradation, with the possibilities multiplying and changing based on regional or tissue-specific locations within the body. Thus, extensive disease-specific research is required into the potential effects of specific eCBs exploitation and modification for therapeutic use [21].

Existing literature highlights potentially promising areas of research, suggesting correlations between the pathogenesis of ASD and the ECS. For example, mutations of neuroligin-3 (a primary protein required for tonic secretion of eCBs) interrupt eCB signaling [34]. Among the effects of such a mutation is the potential for decreased capacity for regulating symptoms of ASDs, such as gastrointestinal function [35-37]. Furthermore, Kerr et al., [38] reported decreased levels of DAGL and MAGL in rats exposed to valproic acid (2-propylpentanoic acid; VPA) Previous studies have found that prenatal exposure to VPA may increase risk of ASD [39] and these findings indicate a possible mechanism by which VPA leads to ASD with respect to altered levels of 2-AG and the corresponding behavioral responses. Additionally, a clinical study completed by Siniscalco et al., [40] demonstrated high expression levels of CB2 in peripheral blood mononuclear cells (PBMCs) of children diagnosed with $\mathrm{ASD}$, indicating the eCBs receptor as a potential target for treatment purposes. Human neuroimaging studies have evaluated the role of the ECS in ASD by measuring responses to social rewards. The studies reported associations between CB1 polymorphisms and ventral striatal cluster activity that suggest a possible link between CB1 polymorphisms and sensitivity to social rewards, a common endophenotype of ASD [41, 42].

\section{Cannabinoids and its therapeutic mechanism}

The mechanism by which cannabinoids could be utilized for treating ASD and its associated disorders, including epilepsy, may possibly be through the synthetic modulation of the ECS, which can help regulate social responses, pleasure, cognition, concentration, body movement, gastrointestinal function, pain, seizures, and the five senses [7, 43]. Unlike THC, CBD is a serotonin (5-hydroxytryptamine) receptor agonist, which is a non-cannabinoid receptor, but may explain the facilitation of the anxiolytic effect [44]. Its antipsychotic effect is attributed to partial agonism at dopamine D2 receptors [45-47], similar to the antipsychotic action of aripiprazole [45]. Additionally, CBD modulates glutamate-GABA systems that may be altered in ASD [48]. Importantly, CBD inhibits the enzyme FAAH that degrades AEA, one of the main endocannabinoids. The modulation of the ECS is primarily targeted to CB1R and $\mathrm{CB} 2 \mathrm{R}$, and synthetic introduction of cannabinoids 
facilitates a process that mimics natural eCBs signaling to affect physiological factors [49]. THC is more effective in binding to CB1R when compared to binding to CB2R [44]. A high density of CB1R can be found in the basal ganglia, hippocampus, neocortex, hypothalamus, and limbic cortex. These neuron terminals affect motor activity, motor coordination, thinking, appetite, and sedation respectively. CB2R can be found on immune cells and tissues, which affect inflammation and immunosuppression [49], as well as the tonsils and spleen, the central nervous system, and in glial and neuronal cells [50]. These interactions, potentiated by cannabinoid treatment, may offer a prospective treatment option for management of ASD related symptoms in the future. Though CB2R is not expressed in neurons under normal conditions, it is highly expressed under pathological conditions (i.e. psychiatric and neurological diseases) [50], and this warrants further investigation. At this time, although still controversial, immune system dysregulation is beginning to receive attention as having a possible role in ASD [51]. The role of CB2R in regulating the immune system and inflammation offers a potentially promising therapeutic mechanism for managing the symptoms associated with ASD etiology [40, 51]. Previous studies have noted an upregulation of CB2R density and an increase in CB2R protein levels in the PBMC of all of the subjects with ASD, while there were no reported differences in CB1R, nor FAAH levels [40]. No significant intragroup variances were also reported for the control group. These results indicate an endocannabinoidCB2 signaling dysregulation in ASD, though CB2R has not shown good cannabinoidergic activity [52]. Despite this, there is a hypothesized treatment opportunity for synthetic eCBs manipulation via $\mathrm{CBD}$ administration. CBD thus, could offer a therapeutic potential for improving motor skill and sleep, while also supporting anxiolytic, antipsychotic [45], and anticonvulsant symptoms [16].

\section{Main text \\ Methodology}

To better understand the current state of evidence regarding the use of cannabinoids among individuals with ASD, we analyzed recently published peerreviewed literature. Our inclusion guidelines required that an article must be written in English (or a translated text is available), published between the years 2000 and 2019, and focus on cannabinoids in the context of autism spectrum disorders. Academic and publicly-available electronic databases, including the Cochrane Library, MEDLINE, Applied Social Services Index and Abstracts, CINAHL, the Education Resources Information Center (ERIC), EMBASE, and PsycINFO were used as sources of literature that fulfilled the predefined inclusion criteria. Search strategies were customized for each database given its use and depth of controlled vocabulary related to the variables of interest, though "cannabinoids" and "autism spectrum disorder" were the most often used search phrases. As such, systematic reviews, reports, and experimental studies were assessed to understand the nature of the evidence, risks, and benefits of cannabis use for ASD.

\section{Findings \\ Clinical trials}

Clinical evidence to evaluate the benefits, risks, and effects of medical cannabis use for those with ASD, have only just begun. A prospective observational study is currently underway at the Children's Hospital of Philadelphia, in collaboration with Zelda Therapeutics (NCT03699527), to create a registry of children with ASD who use medical cannabis, follow their natural history of use, and examine the maximum cannabinoid concentrations in pediatric populations with ASD [53].

Since 2016, three clinical trials examining the effects of medical cannabis on individuals with ASD have been undertaken. As part of a larger study, the effect of a single oral dose of CBD versus placebo on the brain of individuals with and without ASD was compared using magnetic resonance spectroscopy [54]. Recently published results from this clinical trial, indicate that "CBD modulates glutamate-GABA systems, but prefrontalGABA systems respond differently in ASD". As a result, the authors highlight that the effects of a drug tested in a neurotypical population may not generate similar findings in a population with a neurodevelopmental diagnoses [55].

With a focus on behavioral problems in children and youth with ASD, researchers in Jerusalem are studying the efficacy of a cannibinoid mix, while also examining safety and tolerance. The study is a double-blind randomized placebo-controlled trial and the cannabinoid mix consists of a 20:1 ratio in a 160/ $8.0 \mathrm{mg}$ per $\mathrm{ml}$ of $\mathrm{CBD} / \mathrm{THC}$ olive oil-based solution [56]. Results from this clinical trial are eagerly anticipated.

A third study is currently ongoing and examining behavioral effects of cannabidivarin (with weight-based dosing of $10 \mathrm{mg} / \mathrm{kg} /$ day for 12 weeks) versus placebo on children with ASD. The clinical trial is funded by a $\$ 1.3$ million grant from the United States Department of Defense $[57,58]$.

Results from these groundbreaking clinical trials have the potential to help build support for evidence-based recommendations regarding medical cannabis use amongst patients with ASD. Accessing ClinicalTrials.gov is a useful way to follow progress on these studies until 
the time published results are available. However, additional clinical studies which continue to build on existing evidence remain necessary to fully understand the implications of cannabis use in this population.

\section{Preliminary studies}

Thus far, only five research studies to the best of our knowledge exist which have examined the direct effects of medical cannabis in individuals with ASD. The most recently published study conducted in Israel, examined the safety and efficacy of medical cannabis use amongst 188 patients with ASD. Most patients were treated using cannabis oil $(1.5 \%$ THC and $30 \% \mathrm{CBD}$ ), and functional activities of daily living, mood, and quality of life were assessed using structured. Only 93 parents of 155 active participants participated in the six-month follow-up, but a third of participants reported a significant improvement on the three endpoints. Side effects were experienced by approximately $25 \%$ of patients, with the most common side effects reported as restlessness followed by sleepiness and psychoactive effects. This study is limited by the follow-up attrituion at the one and sixmonth follow-up, which was not explained in the publication [59].

In another study also conducted in Israel [8], 53 children with ASD were administered oral cannabinoids under supervision. A 1:20 ratio of CBD and THC was used for a mean duration of 66 days, at a concentration of $30 \%$, with a recommended daily dose of $16 \mathrm{mg} / \mathrm{kg}$ for CBD and $0.8 \mathrm{mg} / \mathrm{kg}$ of THC (maximal daily dose of $600 \mathrm{mg}$ and $40 \mathrm{mg}$ respectively). The study examined changes in the child's comorbid symptoms using prospective bi-weekly interviews with parents. Effects of cannabidiol in respect to hyperactivity, sleep problems, self-injury, and anxiety were reported as an improvement, no change, or worsening. Of interest, changes within the cohort for these symptoms was compared to peer-reviewed data for treatment using conventional methods. As such, hyperactivity was considered improved at $80 \%$, selfinjury at $82 \%$, sleep problems at $60 \%$ and improvement in anxiety symptoms at $64 \%$. Of the children who displayed hyperactivity symptoms, over $68 \%$ reported improvement, over $28 \%$ had no change, while almost 3\% reported worsening of hyperactivity. Improvements in self-injurious behavior were seen in almost $68 \%$ of children, $23.5 \%$ had no change while almost $9 \%$ reported worsening of self-injury. Over $71 \%$ reported improvements in sleep, $23.8 \%$ had no change, while $4.7 \%$ reported worsening effects. Anxiety was improved in over $47 \%$ of children, almost $30 \%$ had no change, while $23.5 \%$ had worse anxiety symptoms. Consequently, the study reported a $74.5 \%$ overall improvement in symptoms of ASD comorbidities, although mild adverse effects of somnolence and decreased appetite were reported in 12 and 6 children respectively. The authors reported no statistically significant difference in hyperactivity, sleep or anxiety of cannabidiol oil compared to conventional treatments of these symptoms. Study limitations, however, include lack of an objective assessment tool and a control group [8].

A third study from Israel focused on children with ASD and severe behavioral concerns and assessed the tolerability and efficacy of cannabidiol-rich cannabis. Led by Dr. Aran at the Shaare-Zedek Medical Center in Jerusalem, as a retrospective feasibility study for their clinical trial grant mentioned earlier (NCT02956226) [56], the study systematically assessed 60 children. Participants were prescribed CBD and THC in a 20:1 ratio, as a whole-plant extract dissolved in olive oil ("mean total daily dose was $3.8 \pm 2.6 \mathrm{mg} / \mathrm{kg} /$ day CBD and $0.29 \pm$ $0.22 \mathrm{mg} / \mathrm{kg} /$ day THC for children who received three daily doses $(n=44)$ and $1.8 \pm 1.6 \mathrm{mg} / \mathrm{kg} /$ day CBD and $0.22 \pm 0.14 \mathrm{mg} / \mathrm{kg} /$ day THC for children who received two daily doses $(n=16) ")$ [60].

The study found $61 \%$ of the behavioral problems among participants were "much improved" or "very much improved" according to parent reports. Improvement was also found in anxiety levels in 39\% of the children and a $47 \%$ improvement in communication. Disruptive behaviors assessed by the Home Situations Questionnaire-Autism Spectrum Disorder [61] and the Autism Parenting Stress Index [62] showed improvement by 29 and 33\% respectively. An additional benefit following cannabis treatment was the reduced intake of medications; $24 \%$ of participants stopped taking medication, over $30 \%$ of children received fewer medications or a lower dose, and $8 \%$ received more additional or a higher dose of their current regimen [60].

Despite the fact that promising outcomes were experienced for participants with ASD, adverse events were reported by 57 parents. These side effects most commonly included hypervigilance, which led to worsening sleep concerns (14\%), irritability (9\%), loss of appetite (9\%), and restlessness (9\%). Other frequently cited adverse events included gastrointestinal symptoms, mood changes, fatigue and unexplained laugh. One serious adverse event was reported, with one participant experiencing a transient psychotic event. The study suggests that strains of medical cannabis with a high THC concentration (6:1-CBD to THC ratio) might increase the likelihood of lead to a psychotic state requiring antipsychotictreatment. The uncontrolled retrospective nature of this study has been cited by the authors as a limitation of this study, in addition to the potential for placebo effects reported in controlled treatment studies in children with ASD, as reported by King et al. [60, 63]. 
A Chilean study published by Kuester et al. [64] examined the effects of cannabis extracts on symptoms of ASD among a small sample of 20 children and one adult with ASD. Participants were monitored after taking sublingual whole plant cannabis extracts for at least 3 months. Almost $72 \%$ of the participants used a balanced THC to CBD extract, $19 \%$ used a high-CBD option, and almost $10 \%$ used high-THC extracts. Details on the administered dosage were not found in the published study or elsewhere; outcomes were assessed using the Clinical Global Impression of Improvement [65] and Autism Parenting Stress Index [62].

Based on these assessments, $66.7 \%$ of the participants showed significant improvement in at least one core ASD symptom like repetitive behaviors, language and social communication. Some improvement was reported by most participants including accepting food, sensory difficulties, seizures, and/or sleep disorders. Despite these reported benefits, three patients reported adverse symptoms: increased agitation $(n=2)$ and irritability $(n=1)$. These conditions were resolved with changes to the cannabis strain [64].

The earliest study identified was of a 6-year old male child with ASD conducted in Austria utilizing Dronabinol (THC). The child received THC dissolved in sesame oil with an initial dosage in the morning constituting one drop $(0.62 \mathrm{mg})$ which gradually increased over the 6 months to the maximum tolerated dose of two drops in the morning, one drop midday and three drops in the evening (total dose of $3.62 \mathrm{mg}$ ). Significant improvements were noted in hyperactivity, irritability, vocal stereotypy and inappropriate speech symptoms, and sterotypic behavior based on assessments using the Aberrant Behavior Checklist [66] at baseline and after six months of treatment. Hyperactivity dropped by 27 points, lethargy decreased by 25 points, irritability by 12 points, stereotypic behavior by 7 points, and inappropriate speech improved by 6 points [67].

\section{Evidence from shared conditions}

Although the aforementioned studies illustrate the potential of cannabis to treat core symptoms of ASD, these studies are constrained in their scope of evidence given their small sample sizes, lack of control groups, and other reported limitations. As such, results from the two clinical trials pending publication of results and completion, and additional large scale clinical trials specific to this population will help build evidence for the safety and efficacy of medical cannabinoids for ASD patients. Until this time, evidence for cannabis use in this population can be merely inferred from studies conducted for pathological conditions shared by other patient populations [68]. However, as noted by Pretzsch et al. [55], the inference and transferability of the effects of cannabis treatments from populations without neurodegenerative conditions on the ASD population are speculative.
Epilepsy An estimated 25\% of children with treatmentresistant epilepsy (who also display other conditions such as mild to severe intellectual disability, sleep disturbances, mood disorders, and psychosis) are comorbid with ASD [69]. Research on the medicinal use of cannabis for treating individuals with seizures and epilepsy have been extensive and as such, seizure disorders are listed as a qualifying condition in states which permit medical cannabis [70]. Gaston and Friedman [71] discuss the therapeutic mechanism of CBD in treating epilepsy, reporting that rather than targeting $\mathrm{CB} 1 \mathrm{R}$ and $\mathrm{CB} 2 \mathrm{R}$, CBD's anticonvulsant properties target "TRPV1, voltage gated potassium and sodium channels, and GPR55, among others" [71].

An Australian survey conducted by Suraev et al., [72] reported that " $15 \%$ of adults with epilepsy and $13 \%$ of parents/guardians of children with epilepsy were currently using, or had previously used, cannabis products to treat epilepsy. Of those with a history of cannabis product use, $90 \%$ of adults and $71 \%$ of parents reported success in reducing seizure frequency after commencing cannabis products."

In an uncontrolled retrospective case study of $272 \mathrm{pa}-$ tients with epilepsy (such as Dravet Syndrome, Rett syndrome, and Lennox-Gastaut syndrome), participants consumed an effective total cannabinoids dose ranging from 0.05 to $9 \mathrm{mg} / \mathrm{kg} /$ day with effective serum levels of CBD ranging from 1.8 to $80 \mathrm{ng} / \mathrm{ml}$. Of the participants, $28 \%$ of subjects experienced a $76-99 \%$ reduction in seizures, $10 \%$ experienced a full clinical response, while $14 \%$ of participants found no effect of artisanal cannabis preparations in reducing seizures. In addition, increased alertness was reported as a desired side effect, while mild and infrequent side effects included decreased appetite, fatigue and somnolence [70].

Substantial interest and willingness to participate in cannabinoid research has offered a long-awaited potential pharmacotherapy solution to treatment-resistant epilepsy and/or limiting the side effects as compared to other treatments [72]. The literature on cannabinoids and epilepsy, specifically for the treatment of intractable seizures in Dravet and Lennox-Gastaut syndromes and co-occurring autism-like behaviors is, as a result, comprehensive [14] and have led to the recent approval as mentioned earlier, of Epidiolex, an oral cannabidiol [19].

Sleep disorders Problems with sleep is a common comorbidity in children and adolescents with ASD, with prevalance estimated between 40 to $80 \%$. Sleep disorders have a significant impact on these individuals, and affects daily life activities, the ability to interact socially, and have also been associated with increased parental stress [73]. A systematic review by Whiting et al. (2015) assessed the benefits and adverse events of cannabinoids 
on several diseases and symptoms such as chronic pain, sleep disorders. The review which included 79 trials and over 6400 participants, concluded that there was lowquality of evidence of the effect of cannabinoids on sleep outcomes [74]. In another systematic review conducted by Gates et al. [75], findings suggested that amongst individuals with a medical condition which may impact sleep, the use of cannabinoids could improve sleep through reduced night-time disturbances. However, amongst studies which utilized objective sleep measures, results of sleep outcomes were inconsistent. In one of the studies examined by Gates et al., a double-blind, placebo-controlled-fourway crossover design assessed the effects of cannabis extracts on memory, early-morning performance, sleep, and sleepiness. The four treatments included: "placebo, $15 \mathrm{mg}$ THC, $5 \mathrm{mg}$ THC combined with $5 \mathrm{mg}$ $\mathrm{CBD}$, and $15 \mathrm{mg}$ THC combined with $15 \mathrm{mg}$ CBD, formulated in 50:50 ethanol to propylene glycol and administered using an oromucosal spray during a 30min period" at night. Results from the study indicated that $15 \mathrm{mg}$ of THC appeared to have sedative effects while $15 \mathrm{mg}$ of $\mathrm{CBD}$ increased alertness [76].

Behavioral deficits An additional core phenotype of ASD is an impaired social functioning ability, including aggression and self-injurious behavior (incidence ranging between 35 and 60\%) [68, 77], which can impair academic achievement, education outcomes, rates of employment, and income [2]. Unfortunately, standard treatments do not benefit approximately $40 \%$ of children with ASD and disruptive behavior, leaving caregivers distressed and increasing social isolation [60]. In a review undertaken by the National Academies of Sciences, Engineering, and Medicine, evidence assessed from systematic reviews and clinical studies indicate limited evidence for the link between cannabis use and social functioning [2].

Psychosocial and mental health Anxiety and mood disorders are also commonly reported to affect those with ASD [68], and at least $40 \%$ are comorbid with anxiety which aggravates other symptoms [16]. In a double-blind randomized study using healthy controls and patients with social anxiety disorder (SAD) with no previous treatment experience, participants received a placebo or a single administration of CBD $(600 \mathrm{mg})$ one and a half hours before a simulated public speaking test. Participants receiving a CBD dose were noted to have decreased "anxiety, cognitive impairment and discomfort in their speech performance as compared to the placebo group" [78].

In two studies evaluated by the National Academies of Sciences, Engineering, and Medicine review [2, 79, 80] data was analyzed from waves 1 and 2 of the National Epidemiologic Survey on Alcohol and Related Conditions $(n=34,653)$. Both studies found no association between cannabis use and anxiety disorders, although both studies reported an association between increased cannabis use with an increased odds of SAD (OR, 1.8; 95\% $\mathrm{CI}=1.1-2.8$ and $\mathrm{OR}, 1.98 ; 95 \% \mathrm{CI}=0.99-6.98$ ). The National Academies of Sciences, Engineering, and Medicine based on their systematic and comprehensive review stated that there is limited evidence for the statistical association between the use of cannabis and the development of any anxiety disorder, with the exception of social anxiety disorders. However, there is moderate evidence to support the association between regular cannabis use and social anxiety disorder.

Although less common, psychosis has also been identified as a comorbidity for ASD [81]. As CBD has been shown to have antipsychotic properties in both human and animal studies, an exploratory double-blind parallelgroup study was conducted to examine the safety and efficacy of CBD in patients with schizophrenia. Randomized patients were to receive CBD $(1000 \mathrm{mg} /$ day $)$ or placebo. If currently prescribed antipsychotic medications, the placebo or CBD was prescribed in addition to the current regiment. CBD may potentially be offered as a new line of treatment for these psychiatric conditions, as "CBD was well tolerated, and rates of adverse events were similar between the CBD and placebo groups" [82]. However, given the adverse outcome of a serious psychotic event discussed earlier in a preliminary study with a patient with ASD [60], the effectiveness of CBD to address psychosis in ASD merits further evaluation.

Effects of cannabinoids on the developing brain of children with and without ASD have also demonstrated the potential for adverse effects such as depressive-like symptoms and an increased risk for psychotic symptoms as an adult $[20,68,83]$. In addition, the impact of cannabis on cognition (specifically, learning, memory, and attention) have also been cited as concerns [2]. Evaluations of studies conducted by the National Academies of Sciences, Engineering, and Medicine illustrate moderate evidence of effects of cannabis on learning, memory, and attention impairment [60, 84], which can impact academic, employment, and social outcomes [2].

Attention-deficit/hyperactivity disorder (ADHD) is also a commonly co-occurring diagnosis in ASD patients with an incidence of 41 to $78 \%$ [8]. ADHD also elevates the risk of substance use disorders in children which could complicate the use of CBD for pharmacotherapy in treating ASDs with co-occurring ADHD [85]. An Australian twin study reported "increased liability to ADHD and elevated autistic traits scores were associated with substance use and misuse," including cannabis use and cannabis use disorders [85]. 
In a six-week, double-blind randomized placebocontrolled trial researchers assessed the effect of a cannabinoid medication (Sativex Oromucosal Spray) in 30 adults with ADHD on cognition. The treatment comprised of a $100-\mu \mathrm{l}$ spray, which contained $2.7 \mathrm{mg}$ THC and $2.5 \mathrm{mg}$ CBD. Improvements were demonstrated in hyperactivity/ impulsivity, inhibition measures, and a non-significant trend suggesting inattention improvement. One serious adverse event related to muscular seizures and spasms was reported [86].

In addition to these comorbidities discussed above, the effect of cannabis should be examined in light of the possibility of medication interactions between cannabis and the various prescription drugs individuals with ASD may be utilizing. Research is lacking regarding dosing regimens [14, 19, 20, 87], which increases the risk of adverse outcomes amongst medical cannabis users.

Toxins such as microbes, heavy metals and pesticides associated with the production of cannabis have also raised concerns. While some studies indicate that CBD has low toxicity in humans and no mutagenic effects [68] other studies suggest toxic contamination may be harmful to the reproductive and developmental system and can cause carcinogenicity and infection [20, 88]. This may be of substantial consideration given the concerns of toxins and its potential association with ASD etiology [89].

\section{Conclusion}

Review of these studies demonstrate the mixed state of evidence with respect to the effects of cannabis on core symptoms of ASD, except for certain types of epilepsy. Given the varying types of studies, populations, cannabis compositions and doses reported in the literature for many shared physiological conditions, the risks and benefits of medical cannabis use in ASD are indirect and insufficient. As a result, medical providers treating individuals with ASD should assess the ethical implications of a cannabis recommendation given the uncertainties associated with its utilization at this time. As such, practitioners should consider behavioral supports accessible to the family and only those pharmacological options which are supported by evidence [90]. Although medical cannabis appears to show promise in addressing core ASD symptoms, evidence-based recommendations are necessary to ensure safety and effectiveness.

Results from the randomized and controlled clinical trials $[54,56,58]$ will help inform effective compositions (cannabinoids, terpenes and flavonoids) of medicinal cannabis to target symptoms and diseases [11] which also recognize "entourage effects" [9]. In time, these studies may help guide future recommendations of cannabis prescriptions for individuals with ASD.
While research on medicinal uses of cannabinoids continues to expand and some co-occurring disorders of ASD such as epilepsy have been evaluated extensively with cannabinoids; equivalent evidence is not currently available to evaluate the efficacy of cannabinoids in treating other multiple conditions associated with ASDs. Given reports suggesting a dysfunctional endocannabinoid system in ASD, the pharmacologic potential of $\mathrm{CBD}$ to impact the symptoms and comorbidities affecting individuals with ASD is significant [43]. Medical cannabis may provide the urgent support needed to address the unique core symptoms of ASD and improving quality of life. Further research, as a result, is necessitated to understand this line of treatment option and to expand the generalizability of results.

\section{Abbreviations}

2-AG: 2-arachidonoylglycerol; ADHD: Attention-deficit/hyperactivity disorder; AEA: Anandamide; ASD: Autism spectrum disorder; CB1: Cannabinoid 1; CB1R: Cannabinoid 1 receptor; CB2: Cannabinoid 2; CB2R: Cannabinoid 2 receptor; CBD: Cannabidiol; DAGL: Diacylglycerol lipase; eCB: Endocannabinoids; ECS: Endocannabinoid system; FAAH: Fatty acid amide hydrolase; FDA: U.S. Food and Drug Administration; FMR1: Fragile X Mental Retardation; MAGL: Monoacylglycerol lipase; SAD: Social anxiety disorder; THC: $\triangle 9$-tetrahydrocannabinol; VPA: Valproic acid; PBMC: Peripheral blood mononuclear cell

\section{Acknowledgements}

Thank you to FIU Embrace for their continued support.

\section{Authors' contributions}

RA, SLB and MM were all involved in the writing and editing of this manuscript. All authors have read and approved the final manuscript.

\section{Funding}

Thank you to FIU Embrace for generously funding the time of all the graduate research assistants on this project. The funding body did not have any role in the design of the study and collection, analysis, and interpretation of data and in writing the manuscript.

\section{Availability of data and materials}

The data supporting the conclusions of this article are available in the databases discussed in the Methodology section and by searching for the specific sources listed in the references.

Ethics approval and consent to participate

Not Applicable

\section{Consent for publication}

Not Applicable

\section{Competing interests}

SLB has received research grants from FIU Embrace, the Florida Department of Health, University of Central Florida / Department of Education, the Dan Marino Foundation, and the Neuroscience Centers of Florida Foundation. RA and MM were funded by FIU Embrace to serve as graduate research assistants.

\section{Author details}

${ }^{1}$ FIU Embrace Florida International University, 11200 SW 8th Street, Miami, FL 33199, USA. 'BRAINN Lab, Florida International University School of Social Work, 11200 SW 8th Street, Miami, FL 33199, USA. ${ }^{3}$ Florida International University School of Public Health Robert Stempel College of Public Health and Social Work Health Promotion and Disease Prevention, 11200 S.W. 8th Street, Miami, FL 33199, USA. ${ }^{4}$ Florida International University School of Social Work Robert Stempel College of Public Health and Social Work, 11200 S.W. 
8th Street, AHC5 585, Miami, FL 33199, USA. ${ }^{5}$ Easterseals Blake Foundation, 750 E Broadway Blvd, Tucson, AZ 85710, USA.

\section{Received: 31 January 2019 Accepted: 26 August 2019 Published online: 29 October 2019}

\section{References}

1. Bridgeman MB, Abazia DT. Medicinal cannabis: history, pharmacology, and implications for the acute care setting. Pharm Ther. 2017;42:180-8.

2. National Academies of Sciences, Engineering, and Medicine. The health effects of cannabis and cannabinoids: the current state of evidence and recommendations for research. Washington, DC: The National Academies Press; 2017. https://doi.org/10.17226/24625.

3. Little B. Why the U.S. made marijuana illegal. History. 2017. https:// www.history.com/news/why-the-u-s-made-marijuana-illegal. Accessed 11 Jan 2019

4. Good CB, Parekh N, Manolis C, Shrank W, Fischer K. Brief commentary: treating pain-the cannabis conundrum. Ann Intern Med. 2019. https://doi. org/10.7326/M18-3237.

5. Gregario J. Physicians, medical marijuana, and the law. Am Med Assoc J Ethics. 2014;16:732-8.

6. Russo EB. Taming THC: potential cannabis synergy and phytocannabinoidterpenoid entourage effects. Br J Pharmacol. 2011;163:1344-64.

7. National Institute on Drug Abuse. Marijuana as medicine. 2017. https:// www.drugabuse.gov/publications/drugfacts/marijuana-medicine. Accessed 3 Jul 2017

8. Barchel D, Stolar O, De-Haan T, Ziv-Baran T, Saban N, Fuchs DO, et al. Oral cannabidiol use in children with autism spectrum disorder to treat related symptoms and co-morbidities. Front Pharmacol. 2019;9. https://doi.org/1 0.3389/fphar.2018.01521.

9. Russo EB. Cannabis and epilepsy: an ancient treatment returns to the fore. Epilepsy Behav. 2017;70:292-7.

10. Nuutinen T. Medicinal properties of terpenes found in cannabis sativa and humulus lupulus. Eur J Med Chem. 2018;157:198-228.

11. Baron EP. Medicinal properties of cannabinoids, terpenes, and flavonoids in cannabis, and benefits in migraine, headache, and pain: an update on current evidence and cannabis science. Headache J Head Face Pain. 2018; 58:1139-86.

12. Marijuana and Cannabinoids. National center for complementary and integrative health. 2018. https://nccih.nih.gov/health/marijuana. Accessed 10 Jan 2019

13. Abrams DI. The therapeutic effects of cannabis and cannabinoids: an update from the national academies of sciences, engineering and medicine report. Eur J Intern Med. 2018;49:7-11.

14. MacCallum CA, Russo EB. Practical considerations in medical cannabis administration and dosing. Eur J Intern Med. 2018:49:12-9.

15. Centers for Disease Control and Prevention. Data and statistics autism spectrum disorder (ASD): Centers for disease control and prevention; 2018. https://www.cdc.gov/ncbddd/autism/data.html. Accessed 11 Jan 2019

16. Gu B. Cannabidiol provides viable treatment opportunity for multiple neurological pathologies of autism spectrum disorder. Glob Drugs Ther. 2017;2:1-4.

17. Siniscalco D. Current findings and research prospective in autism spectrum disorders. Autism. 2013;10:2165.

18. Wei D, Dinh D, Lee D, Li D, Anguren A, Moreno-Sanz G, et al. Enhancement of anandamide-mediated endocannabinoid signaling corrects autismrelated social impairment. Cannabis Cannabinoid Res. 2016;1:81-9.

19. Salgado CA, Castellanos D. Autism spectrum disorder and cannabidiol: have we seen this movie before? Glob Pediatr Health. 2018:5:1-5.

20. Kidd VD. Cannabinoids in the treatment of autism spectrum disorder: demanding data before using fad therapies. Pediatr Neurol. 2018;88:10-1.

21. Pacher $P$, Kunos G. Modulating the endocannabinoid system in human health and disease: successes and failures. FEBS J. 2013;280:1918-43.

22. Chakrabarti B, Persico A, Battista N, Maccarrone M. Endocannabinoid signaling in autism. Neurotherapeutics. 2015;12:837-47.

23. Zhang L, Alger BE. Enhanced endocannabinoid signaling elevates neuronal excitability in fragile X syndrome. J Neurosci. 2010;30:5724-9.

24. Maccarrone M, Rossi S, Bari M, De Chiara V, Rapino C, Musella A, et al. Abnormal mGlu 5 receptor/endocannabinoid coupling in mice lacking FMRP and BC1 RNA. Neuropsychopharmacol. 2010;35:1500-9.
25. Pietropaolo S, Guilleminot A, Martin B, D'Amato FR, Crusio WE. Geneticbackground modulation of core and variable autistic-like symptoms in Fmr1 knock-out mice. PLoS One. 2011;6:e17073.

26. Jung K-M, Sepers M, Henstridge CM, Lassalle O, Neuhofer $\mathrm{D}$, Martin $\mathrm{H}$, et al. Uncoupling of the endocannabinoid signalling complex in a mouse model of fragile X syndrome. Nat Commun. 2012;3:1080.

27. Wang J, Ueda N. Biology of endocannabinoid synthesis system. Prostaglandins Other Lipid Mediat. 2009;89:112-9.

28. Amaral DG, Schumann CM, Nordahl CW. Neuroanatomy of autism. Trends Neurosci. 2008:31:137-45.

29. McFarlane HG, Kusek GK, Yang M, Phoenix JL, Bolivar VJ, Crawley JN. Autism-like behavioral phenotypes in BTBR T+tf/J mice. Genes Brain Behav. 2008:7:152-63.

30. Silverman JL, Tolu SS, Barkan CL, Crawley JN. Repetitive self-grooming behavior in the BTBR mouse model of autism is blocked by the mGluR5 antagonist MPEP. Neuropsychopharmacol. 2010;35:976-89.

31. Onaivi ES, Benno R, Halpern T, Mehanovic M, Schanz N, Sanders C, et al. Consequences of cannabinoid and monoaminergic system disruption in a mouse model of autism spectrum disorders. Curr Neuropharmacol. 2011;9:209-14.

32. McTighe SM, Neal SJ, Lin Q, Hughes ZA, Smith DG. The BTBR mouse model of autism spectrum disorders has learning and attentional impairments and alterations in acetylcholine and kynurenic acid in prefrontal cortex. PLoS One. 2013;8:e62189.

33. Benno R, Smirnova Y, Vera S, Liggett A, Schanz N. Exaggerated responses to stress in the BTBR T+tf/J mouse: an unusual behavioral phenotype. Behav Brain Res. 2009;197:462-5.

34. Földy C, Malenka RC, Südhof TC. Autism-associated neuroligin-3 mutations commonly disrupt tonic endocannabinoid signaling. Neuron. 2013;78:498-509.

35. Di Marzo V. Endocannabinoid signaling in the brain: biosynthetic mechanisms in the limelight. Nat Neurosci. 2011;14:9-15.

36. Li C, Jones PM, Persaud SJ. Role of the endocannabinoid system in food intake, energy homeostasis and regulation of the endocrine pancreas. Pharmacol Ther. 2011;129:307-20.

37. Stone J, Bogner $\mathrm{C}$. The endocannabinoid system as it relates to autism. https:/www.scribd.com/document/218971076/The-EndocannabinoidSystem-as-it-Relates-to-Autism. Accessed 3 Jul 2017.

38. Kerr DM, Downey L, Conboy M, Finn DP, Roche M. Alterations in the endocannabinoid system in the rat valproic acid model of autism. Behav Brain Res. 2013;249:124-32.

39. Roullet FI, Lai JKY, Foster JA. In utero exposure to valproic acid and autism - a current review of clinical and animal studies. Neurotoxicol Teratol. 2013;36:47-56

40. Siniscalco D, Sapone A, Giordano C, Cirillo A, de Magistris L, Rossi F, et al. Cannabinoid receptor type 2, but not type 1 , is up-regulated in peripheral blood mononuclear cells of children affected by autistic disorders. J Autism Dev Disord. 2013:43:2686-95

41. Chakrabarti B, Kent L, Suckling J, Bullmore E, Baron-Cohen S. Variations in the human cannabinoid receptor (CNR1) gene modulate striatal responses to happy faces. Eur J Neurosci. 2006;23:1944-8.

42. Domschke K, Dannlowski U, Ohrmann P, Lawford B, Bauer J, Kugel H, et al. Cannabinoid receptor 1 (CNR1) gene: impact on antidepressant treatment response and emotion processing in major depression. Eur Neuropsychopharmacol. 2008;18:751-9.

43. Schultz S, Siniscalco D. Endocannabinoid system involvement in autism spectrum disorder: an overview with potential therapeutic applications. AIMS Mol Sci. 2019;6:27-37.

44. Russo EB, Burnett A, Hall B, Parker KK. Agonistic properties of cannabidiol at 5-HT1a receptors. Neurochem Res. 2005:30:1037-43.

45. Crippa JA, Zuardi AW, Guimaraes FS. Possible mechanisms involved in the antipsychotic effects of cannabidiol (CBD). Schizophr Bull. 2018;44(Suppl 1):S28.

46. van der Stelt M, Di Marzo V. The endocannabinoid system in the basal ganglia and in the mesolimbic reward system: implications for neurological and psychiatric disorders. Eur J Pharmacol. 2003;480:133-50.

47. Fernández-Ruiz J, Hernández M, Ramos JA. Cannabinoid-dopamine interaction in the pathophysiology and treatment of CNS disorders. CNS Neurosci Ther. 2010;16:e72-91.

48. Busquets-Garcia A, Gomis-González M, Guegan T, Agustín-Pavón C, Pastor A, Mato $S$, et al. Targeting the endocannabinoid system in the treatment of fragile X syndrome. Nat Med. 2013;19:603-7. 
49. Borgelt LM, Franson KL, Nussbaum AM, Wang GS. The pharmacologic and clinical effects of medical cannabis. Pharmacother J Hum Pharmacol Drug Ther. 2013;33:195-209.

50. Chen D, Gao M, Gao F, Su Q, Wu J. Brain cannabinoid receptor 2: expression, function and modulation. Acta Pharmacol Sin. 2017;38:312-6.

51. Siniscalco D, Bradstreet JJ, Cirillo A, Antonucci N. The in vitro GCMAF effects on endocannabinoid system transcriptionomics, receptor formation, and cell activity of autism-derived macrophages. J Neuroinflammation. 2014;11:78.

52. Premoli M, Aria F, Bonini SA, Maccarinelli G, Gianoncelli A, Pina SD, et al. Cannabidiol: recent advances and new insights for neuropsychiatric disorders treatment. Life Sci. 2019;224:120-7.

53. Medical Cannabis Registry and Pharmacology. NIH. U.S. National Library of Medicine. 2018. https://clinicaltrials.gov/ct2/show/NCT03699527. Accessed 4 Jan 2019.

54. Shifting brain excitation-inhibition balance in autism spectrum disorder. $\mathrm{NIH}$. U.S. National Library of Medicine. 2018. https://clinicaltrials.gov/ct2/show/ NCT03537950. Accessed 4 Jan 2019

55. Pretzsch CM, Freyberg J, Voinescu B, Lythgoe D, Horder J, Mendez MA, et al. Effects of cannabidiol on brain excitation and inhibition systems; a randomised placebo-controlled single dose trial during magnetic resonance spectroscopy in adults with and without autism spectrum disorder. Neuropsychopharmacology. 2019;44:1398.

56. Cannabinoids for behavioral problems in children with ASD. NIH. U.S. National Library of Medicine. 2018. https://clinicaltrials.gov/ct2/show/NCT02 956226. Accessed 4 Jan 2019.

57. Arditi L. After other options fail, a family tries medical marijuana for son with autism. Morning Edition. 2018. https://www.npr.org/sections/healthshots/2018/12/25/679830133/after-other-options-fail-a-family-tries-medicalmarijuana-for-son-with-autism. Accessed 4 Jan 2019.

58. Cannabidivarin (CBDV) vs. Placebo in Children With Autism Spectrum Disorder (ASD). NIH. U.S. National Library of Medicine. 2018. https:// clinicaltrials.gov/ct2/show/NCT03202303. Accessed 4 Jan 2019.

59. Bar-Lev Schleider L, Mechoulam R, Saban N, Meiri G, Novack V. Real life experience of medical cannabis treatment in autism: analysis of safety and efficacy. Sci Rep. 2019;9:200.

60. Aran A, Cassuto H, Lubotzky A, Wattad N, Hazan E. Brief report: Cannabidiolrich cannabis in children with autism Spectrum disorder and severe behavioral problems - a retrospective feasibility study. J Autism Dev Disord. 2018. https://doi.org/10.1007/s10803-018-3808-2.

61. Chowdhury M, Aman MG, Lecavalier L, Smith T, Johnson C, Swiezy N, et al. Factor structure and psychometric properties of the revised home situations questionnaire for autism spectrum disorder: the home situations questionnaire-autism Spectrum disorder. Autism Int J Res Pract. 2016;20:528-37.

62. Silva LMT, Schalock M. Autism parenting stress index: initial psychometric evidence. J Autism Dev Disord. 2012;42:566-74.

63. King BH, Dukes K, Donnelly CL, Sikich L, McCracken JT, Scahill L, et al. Baseline factors predicting placebo response to treatment in children and adolescents with autism Spectrum disorders: a multisite randomized clinical trial. JAMA Pediatr. 2013;167:1045-52.

64. Kuester G, Vergara K, Ahumada A, Gazmuri AM. Oral cannabis extracts as a promising treatment for the core symptoms of autism spectrum disorder: preliminary experience in Chilean patients. J Neurol Sci. 2017;381:932-3.

65. Guy W, National Institute of Mental Health (U.S.). Psychopharmacology Research Branch. Division of Extramural Research Programs. ECDEU assessment manual for psychopharmacology. Rockville, Md. : U.S. Dept. of Health, Education, and Welfare, Public Health Service, Alcohol, Drug Abuse and Mental Health Administration, National Institute of Mental Health, Psychopharmacology Research Branch, Division of Extramural Research Programs; 1976. http://archive.org/details/ecdeuassessmentm1933guyw. Accessed 22 Jul 2019.

66. Aman MG, Singh NN, Stewart AW, Field CJ. The aberrant behavior checklist: a behavior rating scale for the assessment of treatment effects. Am J Ment Defic. 1985;89:485-91.

67. Kurz R, Blaas K. Use of dronabinol (delta-9-THC) in autism: a prospective single-case-study with an early infantile autistic child. Cannabinoids. 2010;5:4-6.

68. Poleg S, Golubchik P, Offen D, Weizman A. Cannabidiol as a suggested candidate for treatment of autism spectrum disorder. Prog NeuroPsychopharmacol Biol Psychiatry. 2019;89:90-6.
69. Anderson CL, Evans VF, DeMarse TB, Febo M, Johnson CR, Carney PR. Cannabidiol for the treatment of drug-resistant epilepsy in children: current state of research. J Pediatr Neurol. 2017;15:143-50.

70. Sulak D, Saneto R, Goldstein B. The current status of artisanal cannabis for the treatment of epilepsy in the United States. Epilepsy Behav. 2017; 70:328-33.

71. Gaston TE, Friedman D. Pharmacology of cannabinoids in the treatment of epilepsy. Epilepsy Behav. 2017;70:313-8.

72. Suraev AS, Todd L, Bowen MT, Allsop DJ, McGregor IS, Ireland C, et al. An Australian nationwide survey on medicinal cannabis use for epilepsy: history of antiepileptic drug treatment predicts medicinal cannabis use. Epilepsy Behav. 2017;70:334-40

73. Devnani PA, Hegde AU. Autism and sleep disorders. J Pediatr Neurosci. 2015;10:304-7

74. Whiting PF, Wolff RF, Deshpande S, Di Nisio M, Duffy S, Hernandez AV, et al. Cannabinoids for medical use: a systematic review and meta-analysis. JAMA 2015;313:2456-73.

75. Gates PJ, Albertella L, Copeland J. The effects of cannabinoid administration on sleep: a systematic review of human studies. Sleep Med Rev. 2014;18:477-87.

76. Nicholson AN, Turner C, Stone BM, Robson PJ. Effect of $\Delta-9$ tetrahydrocannabinol and Cannabidiol on nocturnal sleep and earlymorning behavior in young adults. J Clin Psychopharmacol. 2004;24:305-13.

77. Richards C, Moss J, Nelson L, Oliver C. Persistence of self-injurious behaviour in autism spectrum disorder over 3 years: A prospective cohort study of risk markers. J of Neurodev Disord. 2016:8:21.

78. Bergamaschi MM, Queiroz RHC, Chagas MHN, de Oliveira DCG, De Martinis BS, Kapczinski F, et al. Cannabidiol reduces the anxiety induced by simulated public speaking in treatment-Naïve social phobia patients. Neuropsychopharmacology. 2011;36:1219-26.

79. Blanco C, Hasin DS, Wall MM, Flórez-Salamanca L, Hoertel N, Wang S, et al. Cannabis use and risk of psychiatric disorders: prospective evidence from a US national longitudinal study. JAMA Psychiatry. 2016;73:388-95.

80. Feingold D, Weiser M, Rehm J, Lev-Ran S. The association between cannabis use and anxiety disorders: results from a population-based representative sample. Eur Neuropsychopharmacol. 2016;26:493-505.

81. Sagar A, Bishop JR, Tessman DC, Guter S, Martin CL, Cook EH. Cooccurrence of autism, childhood psychosis, and intellectual disability associated with a de novo 3q29 microdeletion. Am J Med Genet A. 2013; 161:845-9.

82. McGuire P, Robson P, Cubala WJ, Vasile D, Morrison PD, Barron R, et al. Cannabidiol (CBD) as an adjunctive therapy in schizophrenia: a multicenter randomized controlled trial. Am J Psychiatry. 2017;175:225-31.

83. Rubino T, Parolaro D. The impact of exposure to cannabinoids in adolescence: insights from animal models. Biol Psychiatry. 2016;79:578-85.

84. D'Souza DC, Ranganathan M. Medical marijuana: is the cart before the horse? JAMA. 2015;313:2431-2.

85. De Alwis D, Agrawal A, Reiersen AM, Constantino JN, Henders A, Martin NG, et al. ADHD symptoms, autistic traits, and substance use and misuse in adult Australian twins. J Stud Alcohol Drugs. 2014;75:211-21.

86. Cooper RE, Williams E, Seegobin S, Tye C, Kuntsi J, Asherson P. Cannabinoids in attention-deficit/hyperactivity disorder: a randomisedcontrolled trial. Eur Neuropsychopharmacol. 2017;27:795-808.

87. Harrison $\mathrm{KL}$, Zane T. Focus on science: is there science behind that?: autism and treatment with marijuana. Sci Autism Treat. 2017;14:12-5.

88. Dryburgh LM, Bolan NS, Grof CPL, Galettis P, Schneider J, Lucas CJ, et al. Cannabis contaminants: sources, distribution, human toxicity and pharmacologic effects. Br J Clin Pharmacol. 2018;84:2468-76.

89. Arora M, Reichenberg A, Willfors C, Austin C, Gennings C, Berggren S, et al. Fetal and postnatal metal dysregulation in autism. Nat Commun. 2017:8:15493.

90. Duvall SW, Lindly O, Zuckerman K, Msall ME, Weddle M. Ethical implications for providers regarding Cannabis use in children with autism Spectrum disorders. Pediatrics. 2019;143:e20180558.

\section{Publisher's Note}

Springer Nature remains neutral with regard to jurisdictional claims in published maps and institutional affiliations. 